\title{
On Heavy-user Bias in A/B Testing
}

\author{
Yu Wang \\ UC Berkeley \\ Berkeley, California, U.S. \\ wang.yu@berkeley.edu
}

\author{
Somit Gupta \\ Microsoft \\ Redmond, Washington, U.S. \\ Somit.Gupta@microsoft.com
}

\author{
Jiannan Lu \\ Microsoft \\ Redmond, Washington, U.S. \\ jiannl@microsoft.com
}

\author{
Ali Mahmoudzadeh \\ Microsoft \\ Redmond, Washington, U.S. \\ Ali.Mahmoudzadeh@microsoft.com
}

\author{
Sophia Liu \\ Microsoft \\ Redmond, Washington, U.S. \\ Sophia.Liu@microsoft.com
}

\begin{abstract}
On-line experimentation (also known as A/B testing) has become an integral part of software development. To timely incorporate user feedback and continuously improve products, many software companies have adopted the culture of agile deployment, requiring online experiments to be conducted and concluded on limited sets of users for a short period. While conceptually efficient, the result observed during the experiment duration can deviate from what is seen after the feature deployment, which makes the A/B test result biased. In this paper, we provide theoretical analysis to show that heavy-users can contribute significantly to the bias, and propose a re-sampling estimator for bias adjustment.
\end{abstract}

\section{CCS CONCEPTS}

- Mathematics of computing $\rightarrow$ Time series analysis;

\section{KEYWORDS}

External validity; jackknife; block bootstrap; causal inference

\section{ACM Reference Format:}

Yu Wang, Somit Gupta, Jiannan Lu, Ali Mahmoudzadeh, and Sophia Liu. 2019. On Heavy-user Bias in A/B Testing. In The 28th ACM International Conference on Information and Knowledge Management (CIKM '19), November 3-7, 2019, Beijing, China. ACM, New York, NY, USA, 4 pages. https://doi. org $/ 10.1145 / 3357384.3358143$

\section{INTRODUCTION}

A/B tests or online controlled experiments are used by a large number of software and technology companies $[5,7]$ to evaluate changes to web services, desktop and mobile applications, and operating systems. In a typical online controlled experiment evaluating a new feature, users are randomly assigned to the treatment group (exposed to the new feature) or the control group (not exposed to the new feature) as they come online to use the product or service. The assignment remains consistent throughout the experiment.

Permission to make digital or hard copies of all or part of this work for personal or classroom use is granted without fee provided that copies are not made or distributed for profit or commercial advantage and that copies bear this notice and the full citation on the first page. Copyrights for components of this work owned by others than the author(s) must be honored. Abstracting with credit is permitted. To copy otherwise, or republish, to post on servers or to redistribute to lists, requires prior specific permission and/or a fee. Request permissions from permissions@acm.org.

CIKM '19, November 3-7, 2019, Beijing, China

() 2019 Copyright held by the owner/author(s). Publication rights licensed to ACM. ACM ISBN 978-1-4503-6976-3/19/11 ..\$15.00

https://doi.org/10.1145/3357384.3358143
During the experiment period, we collect telemetry from all users and compute metric for both groups. We conduct statistical tests to detect differences in metrics values between the treatment and control groups which are unlikely to be observed due to random chance. This establishes a causal relationship between the feature being tested and the measured changes in user behavior $[6,13]$.

One key touchstone of trustworthiness of experimentation is external validity $[1-3,14]$ - can results observed during an experiment period still hold when the new feature being tested is rolled out to the entire user population in the future? There can be multiple factors that affect external validity, such as the novelty effect and the weekday/weekend effect. While such factors are well recognized, there could be other neglected yet common effects that play an important role in determining external validity. In this paper, we highlight heavy-user bias, which describes the phenomenon that frequent users are more likely to be included in an experiment than infrequent ones, rendering the estimated average treatment effect biased. To our best knowledge, it has not been formally discussed in the existing data mining literature, and we hope that this paper can raise the community's awareness on this important issue.

The remainder of the paper is organized as follows. Section 2 discusses the concepts of external validity and heavy user bias in more depth and introduces necessary notations and assumptions. Section 3 derives the closed-form expression of the heavy-user bias, proposes a bias-adjusted estimator based on jackknife [9-12, 18], and illustrates the performance of the bias-adjusted estimator via simulated examples. Section 4 concludes the paper by summarizing the progresses made in this on-going project, and discussing practical challenges and future research directions.

\section{PRELIMINARIES}

\subsection{External validity and heavy user bias}

External validity, also known as generalizability [16], refers to the problem generalizing the findings from the sample units included in the experiment to a larger inference population. External validity is an important problem in causal inference and several papers studied the external validity under a variety of different scenarios such as politics [16] and education [17]. In the context of A/B tests, the external validity of $A / B$ tests could be affected by a variety of factors, such as novelty/primacy effects [15] or weekday/weekend effects.

Heavy user bias is another important yet often overlooked factor that affects the external validity of $\mathrm{A} / \mathrm{B}$ tests. To illustrate what is 
heavy user bias, let us consider a simple example. Suppose a website has two hundred users, half are heavy users who use the website every day, and the other half are light users who use the website with $50 \%$ probability each day. However, if an online experiment is run for just one day, there would be around 150 users using the website. The proportion of heavy users in the experiment sample will be $2 / 3$. In other words, the proportion of heavy users for any A/B test is usually higher than that for the whole population. One simple way to mitigate heavy user bias would be to run the experiment long enough so that the proportion of heavy users and light users remain stable. However, under mild conditions, we show that the heavy user bias is usually inversely proportional to the length of the experiment $k$. This means when the experiment duration doubles, the heavy user bias is only reduced by half. Furthermore, longterm experiments are known to be prone to other critical issues [4]. Therefore, running experiments for longer periods might not be practical.

\subsection{Notations and assumptions}

\begin{tabular}{ll}
\hline Notation & Explanation \\
\hline$Y_{u}(t)$ & the observed outcome of user $u$ at day $t$. \\
$Z_{u}$ & whether the user $u$ is in the treatment group. \\
$R_{u}(t)$ & whether the user $u$ used the product at day $t$. \\
$t_{u}^{0}$ & the first time user $u$ shows up, i.e. $\min \left\{t \mid R_{u}(t)=1\right\}$ \\
$\tau_{u}(t)$ & the treatment effect for user $u$ at day $t$. \\
$c_{u}(t)$ & the control outcome for user $u$ at day $t$. \\
$k$ & duration of the experiment $($ day 1 to day $k)$. \\
\hline
\end{tabular}

The table below describes the notation we will use for the rest of the paper. To derive our theoretical result, we need to make the following assumptions. We will briefly comment why the introduced assumptions are reasonable in our application scenarios, and in Section 4 we will discuss how to further relax the assumptions.

Assumption 1 (stable unit treatment value assumption). One user's outcome is unaffected by other users' treatment assignments. In other words, different users do not interfere with each other.

Assumption 2 (super population). For each user, its behavior can be characterized as a series of triplets $\left\{R_{u}(t), \tau_{u}(t), c_{u}(t)\right\}_{t=1}^{k}$. We assume that this series for each user is an i.i.d. sample from a super population with a probability distribution $\Psi$ :

$$
P\left\{R_{u}(t)=a_{t}, \tau_{u}(t) \leq b_{t}, c_{u}(t) \leq c_{t} ; t=1, \ldots, k\right\},
$$

where $a_{t} \in\{0,1\}, b_{t}, c_{t} \in \mathbb{R}$ for $t \in\{1, \ldots, k\}$.

Assumption 3 (incremental experiment assumption). We assume that for each user $u$, the activity indicator $R_{u}(t)$ is independent of the treatment assignment $Z_{u}$.

Remark 1. Under Assumption 1, the outcome of any user depends only on its own treatment assignment but not other users' treatment assignment. This assumption is reasonable when users do not interact with each other, e.g., users of search engines and operating systems. However, it could break for users that can interact and communicate, such as users of social media. The latter scenarios are beyond the scope of this paper.
Assumption 3 implies that user's visit $R_{u}(t)$ is not affected by whether a user is treated. In other words, we assume our experiment is incremental such that it does not change the frequency of users' visits. This assumption could bring issues if a treatment significantly moves the number of days a user is active (i.e. uses the product). Therefore, it is important to test this assumption before analyzing the data using this framework. One plausible way would be to test whether the average active days per user is the same across treatment and control group. Based on our experience, most experiments do not affect the frequency of users' visits significantly [8].

Under Assumptions 1-3, we can express the observed outcomes of the experimental units as $Y_{u}(t)=R_{u}(t)\left\{Z_{u} \tau_{u}(t)+c_{u}(t)\right\}$, which greatly facilitates the theoretical derivations going forward.

\section{METHODOLOGY}

\subsection{Metric and point estimation}

At the end of an A/B test, we compute metrics to estimate the impact of the treatment on user behavior and make ship decisions. For example, click-through rate (CTR) is a common metric for search engines to measure the effectiveness of online advertisements. In this paper, we focus on the scaled single average

$$
\frac{1}{k} \mathbb{E}\left\{\sum_{t=1}^{k} Y_{u}(t) \mid Z_{u}=z\right\} \quad(z=1,0)
$$

which are arguably the most common metric type in A/B testing ${ }^{1}$. Note that the expectation is calculated with respect to the data generating mechanism in (1), which means it is the average over all the users of the product. For this metric, the average treatment effect (ATE) is the difference between the metric for the treatment group and that for the control group:

$$
\Delta_{\text {scaled }}=\mathbb{E}\left\{\frac{1}{k} \sum_{t=1}^{k} R_{u}(t) \tau_{u}(t)\right\} .
$$

We can estimate $\Delta_{\text {scaled }}$ by the corresponding difference-in-samplemeans derived from the observed data:

$$
\widehat{\Delta}_{\text {scaled }}=\frac{\sum_{u: Z_{u}=1} \sum_{t=1}^{k} Y_{u}(t)}{k N_{T}}-\frac{\sum_{u: Z_{u}=0} \sum_{t=1}^{k} Y_{u}(t)}{k N_{C}},
$$

where $N_{T}=\sum_{u} \mathbf{1}_{t_{u}^{0} \leq k, Z_{u}=1}$ and $N_{C}=\sum_{u} \mathbf{1}_{t_{u}^{0} \leq k, Z_{u}=0}$ are the number of treated/non-treated users that appear during the experiment. They are both random variables.

\subsection{Heavy-user bias}

We define the heavy-user bias of the estimator $\widehat{\Delta}_{\text {scaled }}$ estimating $\Delta_{\text {scaled }}$ as the difference between the expected value of the estimator and the estimand: $\mathbb{E}\left(\widehat{\Delta}_{\text {scaled }}\right)-\Delta_{\text {scaled }}$. Because only users who appear between day $1-k$ are included in the experiment, the expectation of the point estimate of $\widehat{\Delta}_{\text {scaled }}$ is:

$$
\mathbb{E}\left(\widehat{\Delta}_{\text {scaled }}\right)=\mathbb{E}\left\{\frac{1}{k} \sum_{t=1}^{k} \tau_{u}(t) R_{u}(t) \mid t_{u}^{0} \leq k\right\},
$$

The heavy-user bias is a (potentially complex) function of the data generating process in (1). To make the problem somewhat tractable and concrete, we propose a straightforward yet practical

\footnotetext{
${ }^{1}$ We will discuss other types of metrics in Section 4
} 
user behavior model, under which we derive the closed-form expressions of the heavy-user bias. We assume a fixed population, within which there exists user heterogeneity for both heavy and low activity frequencies and outcomes.

Model 1 (Fixed population with user heterogeneity). We use the following model to reflect the heterogeneity on both user activity frequencies and outcomes:

- $R_{u}(t)$ for a user $u$ on day $t$ is i.i.d. from a Bernoulli random variable with success probability $p \sim f(\cdot)$.

- The expectation of the treatment effect for user $u$ is $\mathbb{E} \tau_{u}(t)=$ $\tau(p)$. It implies that the treatment effect could be different for users with different activity parameter $p$ but remains the same across all days.

- Similarly, the expected control outcome of a user is $\mathbb{E} c_{u}(t)=$ $c(p)$.

As demonstrated in the following lemma, Model 1 allows us to derive the closed-form expressions for both $\Delta_{\text {scaled }}$ and $\mathbb{E}\left(\widehat{\Delta}_{\text {scaled }}\right)$, and therefore rigorously quantify the heavy-user bias.

Lemma 1. Under Assumptions 1-3 and Model 1,

$$
\Delta_{\text {scaled }}=\int_{0}^{1} \tau(p) p f(p) d p
$$

and

$$
\mathbb{E}\left(\widehat{\Delta}_{\text {scaled }}\right)=\frac{\int_{0}^{1} \tau(p) p f(p) d p}{\int_{0}^{1} f(p)\left\{1-(1-p)^{k}\right\} d p} .
$$

Proof. First, (5) holds by the definition of Model 1. Second, based on (4),

$$
\begin{aligned}
\mathbb{E}\left(\widehat{\Delta}_{\text {scaled }}\right) & =\mathbb{E}\left\{\tau_{u}(1) R_{u}(1) \mid t_{u}^{0} \leq k\right\} \\
& =\int_{0}^{1} \tau(p) \frac{p}{1-(1-p)^{k}} f\left(p \mid t_{u}^{0} \leq k\right) d p,
\end{aligned}
$$

where $f\left(p \mid t_{u}^{0} \leq k\right)$ is the density of the user activity probability $p$ conditioned on $t_{u}^{0} \leq k$. Using the Bayes' formula, we have

$$
f\left(p \mid t_{u}^{0} \leq k\right)=\frac{f(p) P\left(t_{u}^{0} \leq k \mid p\right)}{\int_{0}^{1} f(p) P\left(t_{u}^{0} \leq k \mid p\right) d p}=\frac{f(p)\left(1-(1-p)^{k}\right)}{\int_{0}^{1} f(p)\left(1-(1-p)^{k}\right) d p} .
$$

By (8) and (9), we complete the proof.

Remark 2. Intuitively, the heavy-user bias arises because light users are less likely to show up during the experiment and therefore are under-represented. If we run the experiment long enough $(k \rightarrow \infty)$, then $\lim _{k \rightarrow \infty} \mathbb{E}\left(\widehat{\Delta}_{\text {scaled }}\right)=\int_{0}^{1} \tau(p) p f(p) d p=\Delta_{\text {scaled. }}$. However, for a finite period $k$, a user with activity probability $p$ has probability $1-$ $(1-p)^{k}$ to show up during the experiment. That shows the proportion of heavy-users during the experiment are higher than that in the whole population.

With the help of Lemma 1, we can now present the main result of this paper.

Proposition 1. Under Assumptions 1-3 and Model 1, if $f(\cdot), \tau(\cdot)$ has gradient and their gradients are continuous, then $\mathbb{E}\left(\widehat{\Delta}_{\text {scaled }}\right)-$ $\Delta_{\text {scaled }}=\Delta_{\text {scaled }} f(0) \cdot k^{-1}+O\left(k^{-2}\right)$.
Proof. Denote $f^{\prime}$ to be $f^{\prime}$ 's gradient. Because $f^{\prime}$ is continuous on the compact set $[0,1], f^{\prime}$ must be uniformly bounded on that set. That implies there exists a positive constant $C>0$ such that $\mid f(p)-$ $f(0) \mid \leq C \cdot p$ for all $p \in[0,1]$. Therefore $\left|\int_{0}^{1} f(p)(1-p)^{k} d p-\int_{0}^{1} f(0)(1-p)^{k} d p\right| \leq$ $\int_{0}^{1} C \cdot p(1-p)^{k} d p$, which implies that

$$
\left|\int_{0}^{1} f(p)(1-p)^{k} d p-\frac{f(0)}{k+1}\right| \leq \frac{1}{(k+1)(k+2)}=O\left(k^{-2}\right) .
$$

By (6) in Lemma 1 and (10), $\mathbb{E}\left\{\widehat{\Delta}_{\text {scaled }}\right\}=\Delta_{\text {scaled }}+f(0) \Delta_{\text {scaled }} k^{-1}+$ $O\left(k^{-2}\right)$, which completes the proof.

Remark 3. When there is no extremely light users $f(0)=0$, it can be seen from the proposition that the first order bias of continuous analysis would be zero. Note that if a user has probability zero of showing up, it will never appear in the experiment. $f(0)$ should be thought of as the limit $\lim _{q \rightarrow 0} P(p \leq q) / q$, which approximately represents users with very light activity. Based on our experience, for many online websites, the proportion of extremely light users is quite significant.

\subsection{Bias-adjusted estimator}

Having derived the heavy-user bias in Proposition 1, we next propose a bias-adjusted estimator to replace the difference-in-means estimator in (3). Our proposal is inspired by jackknife in classical literature $[9,10,18]$, which usually serves as a generic tool to correct first-order biases.

For any fixed experiment duration $k$, let $a$ be the true value of the outcome/metric of interest and $h(k)$ be an estimator of $a$. Assume the the heavy user bias of the estimator can be approximated by a function of $k: h(k)-a=b / k+O\left(k^{-2}\right)$, where $b$ is a parameter. The key insight here is that we can use two points $h(k-1)$ and $h(k)$ to get a better estimate of $a$ with almost no bias of order $O\left(k^{-1}\right)$ : $\widehat{a}=k \cdot h(k)-(k-1) \cdot h(k-1)$. For the scaled single average metric, the natural choice for $h(k)$ is the un-adjusted estimator $\widehat{\Delta}_{\text {scaled }}$. To obtain $h(k-1)$, we can similarly calculate $\widehat{\Delta}_{\text {scaled }}$ using the data of first $k-1$ days. Although this estimate is unbiased, it does not use all the data at hand and can suffer from a large variance. To reduce its variance, we repeat the above procedure by excluding data from day $j=1, \ldots, k$, and average the results. We summarize the above procedure in Algorithm 1.

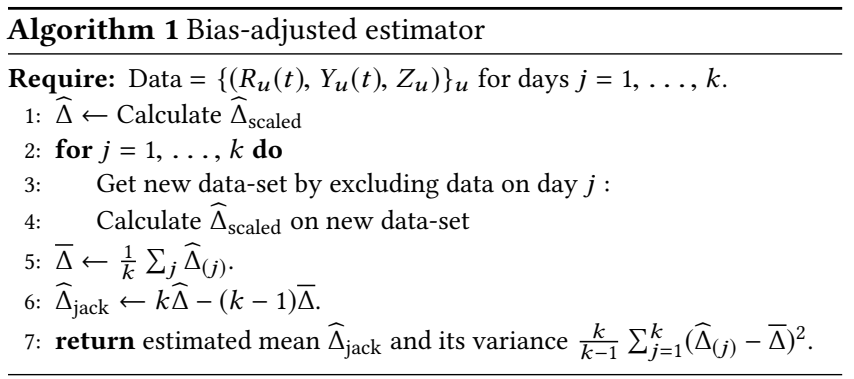

Proposition 2. Under Assumptions 1-3 and Model 1, the heavyuser bias of the bias-adjusted estimator in Algorithm 1 is $\mathbb{E} \widehat{\Delta}_{\text {jack }}-$ $\Delta_{\text {scaled }}=O\left(k^{-2}\right)$. 
Table 1: Average biases and their standard errors

\begin{tabular}{lcc}
\hline & Example 1 & Example 2 \\
\hline Bias of original estimator & $0.0220(0.0023)$ & $0.0373(0.0020)$ \\
Bias of bias-adjusted estimator & $-0.0022(0.0026)$ & $0.0132(0.0023)$ \\
Bias of block-bootstrap estimator & $0.0080(0.0025)$ & $0.0232(0.0022)$ \\
\hline
\end{tabular}

Proof. After excluding day $j$, the remaining data can be viewed as from a $k-1$ day experiment. Thus we apply Proposition 1 to obtain the expectation of $\widehat{\Delta}_{(j)}$, the difference-in-means estimator on data excluding day $j: \mathbb{E} \widehat{\Delta}_{(j)}=\Delta_{\text {scaled }}+\frac{1}{k-1} \Delta_{\text {scaled }} f(0)+O\left(k^{-2}\right)$. Therefore, the expectation of the bias-adjusted estimator is $\mathbb{E} \widehat{\Delta}_{\text {jack }}=$ $k \mathbb{E} \widehat{\Delta}_{\text {scaled }}-\frac{(k-1)}{k} \sum_{j} \widehat{\mathbb{E}}_{(j)}=\Delta_{\text {scaled }}+O\left(k^{-2}\right)$.

\subsection{Simulated examples}

To demonstrate the advantages of the bias-adjusted estimator $\widehat{\triangle}_{\text {jack }}$ in Proposition 2, we present two simulated examples mimicking real-life A/B testing scenarios ${ }^{2}$. For both examples, the experiment lasts for 14 days. The maximal number of units in either treatment or control group is 1000 . Each unit uses the product with probability $p$ for day $t=1, \ldots, k$, where $p$ is generated from $\operatorname{Uniform}(0,1)$. The actual number of units in the treatment/control group varies because some units might not use the product throughout the experiment. The difference between two examples is how we generate the outcomes: if user $u$ uses the product on day $t$, for Example 1 we let $Y_{u}(t)=\left\{\begin{array}{ll}1+p+N\left(0,0.01^{2}\right) & \text { if treated } \\ 1+N\left(0,0.01^{2}\right) & \text { otherwise }\end{array}\right.$ and for Example 2 we let $Y_{u}(t)= \begin{cases}1+\left(1+\frac{1}{10 \cdot U_{u}(t)}\right) \cdot p+N\left(0,0.01^{2}\right) & \text { if treated } \\ 1+N\left(0,0.01^{2}\right) & \text { otherwise }\end{cases}$ where $U_{u}(t)$ is the number of days the user $u$ used the product before day $t$. For both examples, the ground truth $\Delta_{\text {scaled }}=1 / 3$. While Example 1 is strictly under Model 1, Example 2 contains the novelty effect, which violates the assumptions in Model 1. By leveraging the two examples, we aim to examine both the accuracy and the robustness of the proposed bias-adjusted estimator.

For both examples, we repeat the data generation process 100 times. For each simulated data-set, we compute the original differencein-means estimator $\widehat{\Delta}_{\text {scaled }}$, the bias-adjusted estimator $\widehat{\Delta}_{\text {jack }}$, and the block bootstrap estimator[9]. We report the biases and the standard deviations of the three estimators in Table 1. The bias-adjusted estimator produces the smallest bias in both examples. Unfortunately, we do not have an answer why jackknife adjustment works better than bootstrap under our simulated settings.

\section{CONCLUDING REMARKS}

In this paper, we highlighted that the heavy-user bias could affect external validity significantly, and would like to raise the awareness of the data mining community on this issue. We demonstrated that the heavy-user bias exists in A/B testing due to the limited length of an experiment, and proposed a bias-adjusted estimator based on jackknife. Under the fixed population user heterogeneity model, we showed that our estimator could correct the first order heavy-user bias. Simulation studies illustrates the advantages of the proposed methodology.

To conclude the paper, we outline several future research directions to achieve the holy grail of ensuring external validity. First, besides (2) we can consider other types of metrics. For example, double average: $\Delta_{\text {double }}=\mathbb{E}\left\{\frac{\sum_{t=1}^{k} R_{u}(t) \tau_{u}(t)}{\sum_{t=1}^{k} R_{u}(t)}\right\}$. Second, it is desirable to evaluate the bias-adjusted estimator in some empirical data-sets. Third, it is important to understand the joint effect of multiple factors that affect external validity. The simulated examples showed that our proposed estimator provided a more accurate estimate in the presence of both the heavy user bias and the novelty effect. However, we might need to consider other possible effects in practice. Last but not least, it would be interesting to extend the current study to the network setting with user interference.

\section{REFERENCES}

[1] Donald T. Campbell. 1957. Factors relevant to the validity of experiments in social settings. Psychological Bulletin 54 (1957), 297-312.

[2] Donald T Campbell and Julian C Stanley. 1963. Experimental and Quasiexperimental Designs for Research. Cengage Learning, New York, NY.

[3] Thomas D Cook, Donald Thomas Campbell, and William Shadish. 2002. Experimental and Quasi-experimental Designs for Generalized Causal Inference. Houghton Mifflin Boston, Boston, MA.

[4] P. Dmitriev, B. Frasca, S. Gupta, R. Kohavi, and G. Vaz. 2016. Pitfalls of long-term online controlled experiments. In 2016 IEEE International Conference on Big Data (Big Data). 1367-1376. https://doi.org/10.1109/BigData.2016.7840744

[5] Henning Hohnhold, Deirdre O'Brien, and Diane Tang. 2015. Focusing on the longterm: It's good for users and business. In Proceedings of the 21th ACM SIGKDD International Conference on Knowledge Discovery and Data Mining. ACM, Sydney, Australia, 1849-1858.

[6] Guido W Imbens and Donald B Rubin. 2015. Causal inference in statistics, social, and biomedical sciences. Cambridge University Press, New York, NY.

[7] Eugene Kharitonov, Alexey Drutsa, and Pavel Serdyukov. 2017. Learning sensitive combinations of A/B test metrics. In Proceedings of the Tenth ACM International Conference on Web Search and Data Mining. ACM, Cambridge, UK, 651-659.

[8] Ron Kohavi, Alex Deng, Roger Longbotham, and Ya Xu. 2014. Seven rules of thumb for web site experimenters. In Proceedings of the 20th ACM SIGKDD international conference on Knowledge discovery and data mining. ACM, New York, NY, 1857-1866.

[9] Hans R. Kunsch. 1989. The Jackknife and the Bootstrap for general stationary observations. Annals of Statistics 17, 3 (1989), 1217-1241.

[10] Rupert G. Miller. 1974. The jackknife: A review. Biometrika 61, 1 (1974), 1-15.

[11] M. H. Quenouille. 1956. Notes on bias in estimation. Biometrika 43 (1956), 353.

[12] J N K Rao. 1965. A note on estimation of ratios by Quenouille's method. Biometrika 52, 3 (1965), 647-649.

[13] Donald B. Rubin. 2008. For objective causal inference, design trumps analysis. The Annals of Applied Statistics 2 (2008), 808-840.

[14] Arman Sabbaghi and Qiang Huang. 2018. Model transfer across additive manufacturing processes via mean effect equivalence of lurking variables. Annals of Applied Statistics 12 (2018), 2409-2429.

[15] Daniel A. Sheinin, Sajeev Varki, and Christy Ashley. 2011. The differential effect of Ad novelty and message usefulness on brand judgments. Fournal of Advertising 40, 3 (2011), 5-18.

[16] Elizabeth A. Stuart, Stephen R. Cole, Catherine P. Bradshaw, and Philip J. Leaf. 2011. The use of propensity scores to assess the generalizability of results from randomized trials. Fournal of the Royal Statistical Society, Series A 174, 2 (2011), 369-386.

[17] Elizabeth Tipton, Larry Hedges, Michael Vaden-Kiernan, Geoffrey Borman, Kate Sullivan, and Sarah Caverly. 2014. Sample selection in randomized experiments: A new method using propensity score stratified sampling. Fournal of Research on Educational Effectiveness 7, 1 (2014), 114-135.

[18] John W Tukey. 1958. Bias and confidence in not quite large samples. Annals of Mathematical Statistics 29 (1958), 614. 